\title{
QUIVER MUTATION SEQUENCES AND $q$-BINOMIAL IDENTITIES
}

\author{
AKISHI KATO, YUMA MIZUNO, AND YUJI TERASHIMA
}

\begin{abstract}
In this paper, first we introduce a quantity called a partition function for a quiver mutation sequence. The partition function is a generating function whose weight is a $q$-binomial associated with each mutation. Then, we show that the partition function can be expressed as a ratio of products of quantum dilogarithms. This provides a systematic way of constructing various $q$-binomial multisum identities.
\end{abstract}

\section{Contents}

1. Introduction

2. Quiver mutation sequences

2.1. Quivers and mutation sequences

2.2. Ice quivers and $c$-vectors

2.3. Green and red mutations

2.4. Noncommutative algebra $\widehat{\mathbb{A}}_{Q}$

3. Partition functions

4. Main Results

4.1. Statement of the main results

4.2. Proof of Theorem 4.1

4.3. Proof of Theorem 4.2

5. Examples

References

\section{INTRODUCTION}

Quiver mutations appear in various areas of mathematics and physics such as Donaldson-Thomas theory, low-dimensional topology, representation theory, and gauge theory. It is very important to capture quantitatively a common structure hidden in various guises of quiver mutations.

In our previous works 9, 10, we introduced the partition q-series for a quiver mutation loop - a mutation sequence whose final quiver is isomorphic to the initial one. The partition $q$-series is defined as a sum over state variables defined on a graph which describes the sequential evolution of the quiver, and depend only on the combinatorial structures of quiver mutation sequences. For certain class of quiver mutation sequences called reddening sequences, we proved that the graded version of partition $q$-series essentially coincides with the combinatorial DonaldsonThomas invariants introduced by Keller [11, 12, 13, motivated by Nagao [17, 18] and Reineke 22, 23. This is a combinatorial version of more general invariants 
studied in Kontsevich and Soibelman's groundbreaking work [15, 16, on the BPS state counting problem. It goes without saying that cluster algebras play crucial role in the development such as the periodicity of $T$ - and $Y$-systems and the associated dilogarithm identities [4, 8, 14, 19, 20.

In this paper, we first introduce a quantity called a partition function for a quiver mutation sequence. Just like the partition $q$-series, the partition functions are defined as a sum over states on a graph generated by the sequential evolution of the quiver. It has a $\mathbb{Z}^{n}$-grading which comes from $c$-vectors, as is the case with the partition $q$-series. The partition function is a generating function whose coefficients are a product of local weights associated with mutations. As compared with the partition $q$-series, however, there are some important differences. (1) The partition functions are defined for mutation sequences rather than mutation loops. (2) The partition functions depend explicitly on initial conditions on state variables. (3) The partition functions uses q-binomial coefficients as the local weights.

We then show that the partition function is an "invariant of quiver mutation sequences" in the following sense: if two mutation sequences $\boldsymbol{m}$ and $\boldsymbol{m}^{\prime}$ on a quiver $Q$ result in same quivers and $c$-vectors, then the two partition functions associated to them coincide, that is, $Z_{m}=Z_{m^{\prime}}$. This is considered to be a $q$-binomial version of so-called quantum dilogarithm identities. In fact, we show that the partition function can be expressed as a ratio of products of quantum dilogarithms. Then, we can use the usual quantum dilogarithm identities to show the invariance of the partition function. These results provide a systematic way of constructing various $q$-binomial multisum identities.

This paper is organized as follows. In Section 2 we review some definitions and terminologies about quiver mutations. In Section 3 we define the partition functions of mutation sequences. We then give our main results in Section 4 . We give a formula that express the partition function as a ratio of products of quantum dilogarithms, then we prove the identities of the partition functions. In Section 5 we give some examples of the partition functions of mutation sequences, which describe our main results in concrete situations.

Acknowledgments. We would like to thank R. Inoue, R. Kobayashi, A. Kuniba, and M. Yamazaki for valuable discussions. This work is partially supported by JSPS KAKENHI Grant Number JP16K13752, JP16H03931 and 25400083, and by CREST, JST.

\section{Quiver mutation SEQUences}

2.1. Quivers and mutation sequences. We briefly review the notion of quiver mutations introduced by Fomin and Zelevinsky [3].

A quiver $Q$ is an oriented graph. In this paper we assume that all quivers are finite oriented graphs without loops or 2-cycles:

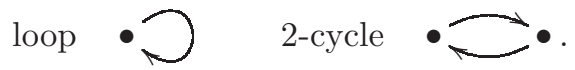

Throughout the paper, we identify the set of vertices of $Q$ with $\{1,2, \ldots, n\}$. Let $Q_{i j} \in \mathbb{N}=\{0,1,2, \cdots\}$ be a multiplicity of the arrows, and consider it as $(i, j)$ entry of an $n \times n$ matrix. Then the quiver can be identified with a skew-symmetric integer $n \times n$ matrix $B$ given by

$$
B_{i j}=Q_{i j}-Q_{j i}
$$


Let $k$ be a vertex in a quiver $Q$. The mutation $\mu_{k}$ is a transformation of the quiver $Q$ to another quiver $\mu_{k}(Q)$ as defined below. The mutated quiver $\mu_{k}(Q)$ has the same vertices as $Q$. Its arrows are obtained from those of $Q$ via three steps:

1) for each length two path $i \rightarrow k \rightarrow j$, add a new arrow $i \rightarrow j$;

2) reverse the direction of all arrows with source or target $k$;

3) remove all 2-cycles which arose in step 1.

Mutations are involutive, i.e. $\mu_{k}\left(\mu_{k}(Q)\right)=Q$ for any $1 \leq k \leq n$. The mutations can be seen as a transformation of skew-symmetric matrices. Let $B$ be the skewsymmetric corresponding to the quiver $Q$. Then the skew-symmetric matrix $\mu_{k}(B)$ corresponding to $\mu_{k}(Q)$ is given by

$$
\mu_{k}(B)_{i j}= \begin{cases}-B_{i j} & \text { if } i=k \text { or } j=k \\ B_{i j}+\operatorname{sgn}\left(B_{i k}\right) \max \left(B_{i k} B_{k j}, 0\right) & \text { otherwise. }\end{cases}
$$

A mutation sequence of a quiver $Q$ is a finite sequence of mutations starting from $Q$. We denote it by $\boldsymbol{m}=\left(m_{1}, m_{2}, \ldots, m_{T}\right)$, a finite sequence of vertices of $Q$. The mutation sequence $\boldsymbol{m}$ induces a (discrete) time evolution of the quivers:

$$
Q(0) \stackrel{\mu_{m_{1}}}{\longrightarrow} Q(1) \stackrel{\mu_{m_{2}}}{\longrightarrow} \cdots \longrightarrow Q(t-1) \stackrel{\mu_{m_{t}}}{\longrightarrow} Q(t) \longrightarrow \cdots \stackrel{\mu_{m_{T}}}{\longrightarrow} Q(T),
$$

where $Q(t):=\mu_{m_{t}}(Q(t-1))$. The quiver $Q(0)$ is called the initial quiver and $Q(T)$ the final quiver. We will use the notation $\mu_{\boldsymbol{m}}(Q)=\mu_{m_{T}}\left(\cdots \mu_{m_{2}}\left(\mu_{m_{1}}(Q)\right) \cdots\right)$.

2.2. Ice quivers and $c$-vectors. We will follow the terminology in [1. An ice quiver is a pair $(\widetilde{Q}, F)$ where $\widetilde{Q}$ is a quiver and $F$ is a (possibly empty) subset of vertices of $\widetilde{Q}$ such that there are no arrows between them. Vertices in $F$ are called frozen vertices. Two ice quivers $(\widetilde{Q}, F)$ and $\left(\widetilde{Q}^{\prime}, F^{\prime}\right)$ are frozen isomorphic if $F=F^{\prime}$ and there is an isomorphism of quivers $\phi: \widetilde{Q} \rightarrow \widetilde{Q}^{\prime}$ such that $\phi \mid F$ is an identity map.

For any quiver $Q$, we can canonically construct an ice quiver $Q^{\wedge}$ called a framed quiver. This is obtained from $Q$ by adding, for each vertex $i$, a new frozen vertex $i^{\prime}$ and a new arrow $i \rightarrow i^{\prime}$.

Let $\boldsymbol{m}=\left(m_{1}, m_{2}, \ldots, m_{T}\right)$ be a mutation sequence of $Q$. By putting

$$
\widetilde{Q}(0)=Q^{\wedge}, \quad \widetilde{Q}(t)=\mu_{m_{t}}(\widetilde{Q}(t-1)) \quad(t=1,2, \ldots, T)
$$

we can construct a sequence of ice quivers

$$
\widetilde{Q}(0) \stackrel{\mu_{m_{1}}}{\longrightarrow} \widetilde{Q}(1) \stackrel{\mu_{m_{2}}}{\longrightarrow} \cdots \longrightarrow \widetilde{Q}(t-1) \stackrel{\mu_{m_{t}}}{\longrightarrow} \widetilde{Q}(t) \longrightarrow \cdots \stackrel{\mu_{m_{T}}}{\longrightarrow} \widetilde{Q}(T) .
$$

Note that we never mutate at frozen vertices $F=\left\{1^{\prime}, \ldots, n^{\prime}\right\}$. Let $\widetilde{B}(t)$ denote the skew-symmetric matrix corresponding to $\widetilde{Q}(t)$.

The $c$-vectors, which were introduced by Fomin and Zelevinsky [5], are defined by counting arrows to/from frozen vertices.

Definition 2.1. A c-vector of a vertex $v$ in $Q(t)$ is a vector in $\mathbb{Z}^{n}$ given by

$$
c_{v}(t):=\left(\widetilde{B}(t)_{v i^{\prime}}\right)_{i=1}^{n} .
$$




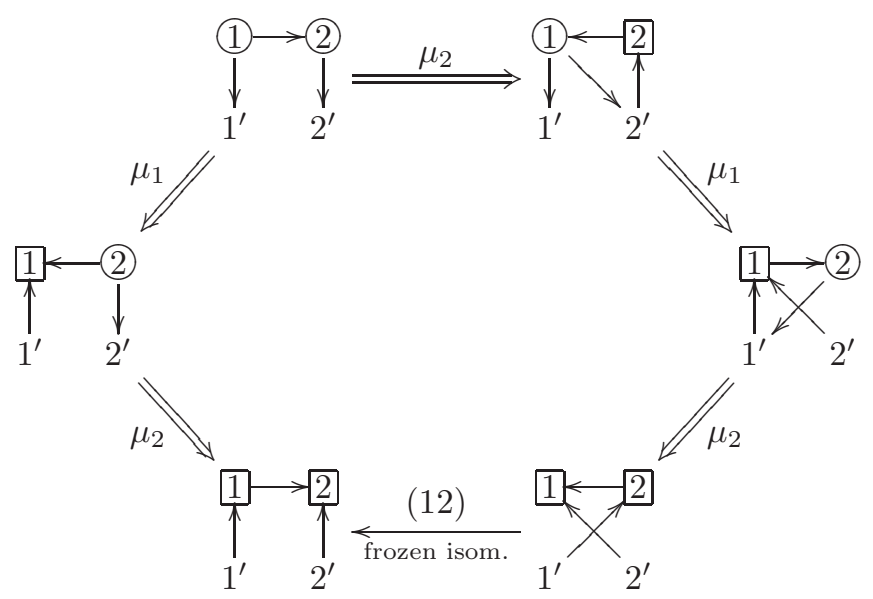

Figure 1. Pentagon and the $A_{2}$ quiver. The green and red vertices are marked with circles and boxes, respectively. Both $\boldsymbol{m}=(1,2)$ and $\boldsymbol{m}^{\prime}=(2,1,2)$ are maximal green sequences.

If the vertices of $\widetilde{Q}(t)$ are ordered as $\left(1, \ldots, n, 1^{\prime}, \ldots, n^{\prime}\right)$, the skew-symmetric matrix $\widetilde{B}(t)$ has the block form

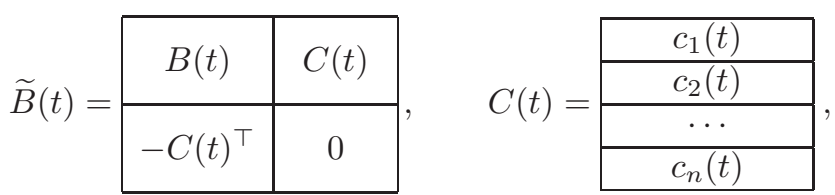

where $X^{\top}$ denotes the transpose of $X$. The $n \times n$ block $C(t)$ is called c-matrix, which consists of row of $c$-vectors. By construction, $c_{i}(0)=e_{i}$, where $e_{i}$ is the $i$-th standard unit vector in $\mathbb{Z}^{n}$.

2.3. Green and red mutations. The vertex $v$ of $Q(t)$ is called green (resp. red) if $c_{v}(t) \in \mathbb{N}^{n}$ (resp. $-c_{v}(t) \in \mathbb{N}^{n}$ ). Note that every vertex of the initial quiver $Q(0)$ is green by construction. The following crucial property is satisfied by $c$-vectors:

Theorem 2.2 (Sign coherence [2, 21, 18]). Any vertex $v$ in $Q(t)$ is either green or red.

The mutation $\mu_{m_{t}}: Q(t-1) \rightarrow Q(t)$ is green (resp. red) if the mutating vertex $m_{t}$ is green (resp. red) on $Q(t-1)$, i.e. on the quiver before mutation. The sign $\varepsilon_{t}$ of the mutation $\mu_{m_{t}}$ is defined by

$$
\varepsilon_{t}= \begin{cases}+1 & \text { if } \mu_{m_{t}} \text { is green } \\ -1 & \text { if } \mu_{m_{t}} \text { is red. }\end{cases}
$$

A mutation sequence $\boldsymbol{m}=\left(m_{1}, m_{2}, \ldots, m_{T}\right)$ is called a green sequence if $m_{t}$ is green for all $t$. A mutation sequence $\boldsymbol{m}$ is called a reddening sequence if all vertices of the final quiver $Q(T)$ are red. A mutation sequence $\boldsymbol{m}$ is called a maximal green sequence if it is green and reddening. All maximal green sequences are reddening by definition, but there is a reddening sequence that is not maximal green (see 
Example 5.5). In Figure 1, the two maximal green sequences $(1,2)$ and $(2,1,2)$ are shown for the $A_{2}$ quiver.

2.4. Noncommutative algebra $\widehat{\mathbb{A}}_{Q}$. We introduce a noncommutative associative algebra which we use in the definition of the partition function.

Let $Q$ be a quiver with vertices $\{1,2, \ldots, n\}$. We define a skew symmetric bilinear form $\langle\rangle:, \mathbb{Z}^{n} \times \mathbb{Z}^{n} \rightarrow \mathbb{Z}$ by

$$
\left\langle e_{i}, e_{j}\right\rangle=B_{i j}=-B_{j i}=Q_{i j}-Q_{j i},
$$

where $e_{1}, \ldots, e_{n}$ are the standard basis vectors in $\mathbb{Z}^{n}$.

Let $\mathbb{A}_{Q}$ be a noncommutative associative algebra over $\mathbb{Q}\left(q^{1 / 2}\right)$ presented as

$$
\mathbb{A}_{Q}=\mathbb{Q}\left(q^{\frac{1}{2}}\right)\left\langle y^{\alpha}, \alpha \in \mathbb{N}^{n} \mid y^{\alpha} y^{\beta}=q^{\frac{1}{2}\langle\alpha, \beta\rangle} y^{\alpha+\beta}\right\rangle .
$$

Its completion with respect to the $\mathbb{N}^{n}$-grading is denoted by $\widehat{\mathbb{A}}_{Q}$, which can be regarded as the ring of noncommutative power series in $y_{i}:=y^{e_{i}}(i=1, \ldots, n)$.

We will often use the following relations $\left(\alpha=\left(\alpha_{1}, \ldots, \alpha_{n}\right) \in \mathbb{Z}^{n}\right)$ :

$$
\begin{aligned}
& y_{1}^{\alpha_{1}} y_{2}^{\alpha_{2}} \ldots y_{n}^{\alpha_{n}}=q^{\frac{1}{2} \sum_{i<j} B_{i j} \alpha_{i} \alpha_{j}} y^{\alpha}, \\
& y^{\alpha}=q^{-\frac{1}{2} \sum_{i<j} B_{i j} \alpha_{i} \alpha_{j}} y_{1}^{\alpha_{1}} y_{2}^{\alpha_{2}} \ldots y_{n}^{\alpha_{n}} .
\end{aligned}
$$

The $q$-factors in the definition of $y^{\alpha}$ are chosen to guarantee invariance under the reindexing of the $y_{i}$ 's.

\section{PARTition FunCtions}

In this section, we introduce partition functions for mutation sequences.

Let $Q$ be a quiver with vertices $\{1,2, \ldots, n\}$. We consider a mutation sequence $\boldsymbol{m}=\left(m_{1}, m_{2}, \ldots, m_{T}\right)$ of $Q$ :

$$
Q(0) \stackrel{\mu_{m_{1}}}{\longrightarrow} Q(1) \stackrel{\mu_{m_{2}}}{\longrightarrow} \cdots \longrightarrow Q(t-1) \stackrel{\mu_{m_{t}}}{\longrightarrow} Q(t) \longrightarrow \cdots \stackrel{\mu_{m_{T}}}{\longrightarrow} Q(T) .
$$

We first give a family of $s$-variables $\left\{s_{i}\right\}$, $k$-variables $\left\{k_{t}\right\}$, and $k^{\vee}$-variables $\left\{k_{t}^{\vee}\right\}$ by the following rule:

(i) An "initial" $s$-variable $s_{v}$ is attached to each vertex $v$ of the initial quiver $Q=Q(0)$.

(ii) When we mutate a quiver at vertex $v$, we add a "new" $s$-variable associated with $v$.

(iii) We associate $k_{t}$ and $k_{t}^{\vee}$ with each mutation at $m_{t}$.

The $s^{-}, k^{-}$, and $k^{\vee}$-variables are not regarded as independent. We require a linear relation for each mutation. If the quiver $Q(t-1)$ equipped with $s$-variables $\left\{s_{i}\right\}$ is mutated at vertex $v=m_{t}$ to give $Q(t)$, then $k$ - and $s$-variables are required to satisfy

$$
k_{t}= \begin{cases}s_{v}+s_{v}^{\prime}-\sum_{a \rightarrow v} s_{a} & \text { if } \mu_{v} \text { is green }\left(\varepsilon_{t}=1\right) \\ \sum_{v \rightarrow b} s_{b}-\left(s_{v}+s_{v}^{\prime}\right) & \text { if } \mu_{v} \text { is red }\left(\varepsilon_{t}=-1\right)\end{cases}
$$


Here, $s_{v}^{\prime}$ is the "new" $s$-variable attached to mutated vertex $v$, and the sum is over all the arrows of $Q(t-1)$. Similarly, $k^{\vee}$ - and $s$-variables satisfy

$$
k_{t}^{\vee}= \begin{cases}\sum_{v \rightarrow b} s_{b}-\left(s_{v}+s_{v}^{\prime}\right) & \text { if } \mu_{v} \text { is green }\left(\varepsilon_{t}=1\right) \\ s_{v}+s_{v}^{\prime}-\sum_{a \rightarrow v} s_{a} & \text { if } \mu_{v} \text { is red }\left(\varepsilon_{t}=-1\right)\end{cases}
$$

Therefore,

$$
k_{t}+k_{t}^{\vee}=\sum_{v \rightarrow b} s_{b}-\sum_{a \rightarrow v} s_{a}
$$

holds at each mutation.

It is sometimes useful to view the relation (3.2) as a discrete time evolution of $s$-variables with the control parameters $\left\{k_{t}\right\}$. Let $s_{i}(t)$ be the $s$-variable associated with the vertex $i$ in $Q(t)$. Then (3.2) can be written as

$$
s_{i}(t)= \begin{cases}s_{i}(t-1) & \text { if } i \neq v \\ k_{t}-s_{v}(t-1)+\sum_{a} Q(t)_{a, v} s_{a}(t-1) & \text { if } i=v \text { and } \mu_{v} \text { is green } \\ -k_{t}-s_{v}(t-1)+\sum_{b} Q(t)_{v, b} s_{b}(t-1) & \text { if } i=v \text { and } \mu_{v} \text { is red. }\end{cases}
$$

With this notation, (3.4) reads as

$$
k_{t}+k_{t}^{\vee}=\sum_{i} B(t-1)_{v, i} s_{i}(t-1)=-\sum_{i} B(t-1)_{i, v} s_{i}(t-1) .
$$

Consequently, we have

Proposition 3.1. Each $k^{\vee}$-variable is written as a $\mathbb{Z}$-linear combination of $k$ variables and initial $s$-variables.

Hereafter, we write $r_{i}$ for the initial $s$-variables $s_{i}(0)$ at the vertex $i$ of $Q$ for $i \in\{1,2, \ldots, n\}$.

We now introduce a mutation weight.

Definition 3.2. Consider a mutation $\mu_{v}$ at the vertex $v$. Then the mutation weight of $\mu_{k}$ is given by

$$
W^{\varepsilon}\left(k, k^{\vee}\right)=q^{-\frac{1}{2} \varepsilon k k^{\vee}}\left[\begin{array}{c}
k+k^{\vee} \\
k
\end{array}\right]_{q^{\varepsilon}} .
$$

Here, $\varepsilon \in\{1,-1\}$ is the sign of $\mu_{v}$ and $k \in \mathbb{N}, k^{\vee} \in \mathbb{Z}$ are $k$-, $k^{\vee}$-variables associated with the mutation $\mu_{v}$, respectively. The $q$-binomial coefficient is defined by

$$
\left[\begin{array}{c}
m \\
k
\end{array}\right]_{q}=\frac{\left(q^{m-k+1} ; q\right)_{k}}{(q ; q)_{k}}
$$

with $(x ; q)_{k}:=\prod_{i=0}^{k-1}\left(1-x q^{i}\right)$.

Remark 3.3. $W^{\varepsilon}\left(k, k^{\vee}\right)$ is a Laurent polynomial of $q^{1 / 2}$. Note that we allow $k^{\vee}$ to be negative integer since our definition of $q$-binomial coefficient (3.8) is valid even if $m<k$. 
Let $\alpha_{t} \in \mathbb{N}^{n} \backslash\{0\}$ denote the sign-corrected c-vector

$$
\alpha_{t}=\varepsilon_{t} c_{m_{t}}(t-1)
$$

namely, the product of the sign and the $c$-vector of the vertex on which the $t$-th mutation takes place.

Definition 3.4. Consider a quiver mutation sequence $\boldsymbol{m}=\left(m_{1}, \cdots, m_{T}\right)$ with an initial quiver $Q$. The partition function of $\boldsymbol{m}$ with initial $s$-variables $r=$ $\left(r_{1}, \ldots, r_{n}\right) \in \mathbb{Z}^{n}$ is given by

$$
Z_{\boldsymbol{m}}(r)=\sum_{k \geq 0}\left(\prod_{t=1}^{T} W^{\varepsilon_{t}}\left(k_{t}, k_{t}^{\vee}\right)\right) y^{\sum_{t=1}^{T} k_{t} \alpha_{t}} \in \widehat{\mathbb{A}}_{Q}
$$

Here the sum is taken over $k=\left(k_{1}, \ldots, k_{T}\right) \in \mathbb{N}^{T}$, and all the mutation weights $\left\{W^{\varepsilon_{t}}\left(k_{t}, k_{t}^{\vee}\right)\right\}_{1 \leq t \leq T}$ are considered as functions of $k \in \mathbb{N}^{T}$ and initial $s$-variables $r \in \mathbb{Z}^{n}$ via Proposition 3.1. Consequently, $Z_{\boldsymbol{m}}$ defines a map $Z_{\boldsymbol{m}}: \mathbb{Z}^{n} \rightarrow \widehat{\mathbb{A}}_{Q}$, $r \mapsto Z_{m}(r)$.

Example 3.5. Let us consider the $A_{2}$ quiver

$$
Q=1 \longrightarrow 2
$$

and a mutation sequence $\boldsymbol{m}=(1,2)$. See the left half of Figure 11. We with to compute the partition function $Z_{\boldsymbol{m}}\left(r_{1}, r_{2}\right)$. The sign-corrected $c$-vector $\alpha_{t}$ and the $\operatorname{sign} \varepsilon_{t}$ are computed to be

$$
\begin{array}{ll}
\alpha_{1}=(1,0), & \varepsilon_{1}=+, \\
\alpha_{2}=(0,1), & \varepsilon_{2}=+.
\end{array}
$$

The $s$-variables change as follows (cf. (3.5)):

\begin{tabular}{c|c|c} 
& $s_{1}(t)$ & $s_{2}(t)$ \\
\hline$t=0$ & $r_{1}$ & $r_{2}$ \\
$t=1$ & $k_{1}-r_{1}$ & $r_{2}$ \\
$t=2$ & $k_{1}-r_{1}$ & $k_{2}-r_{2}$
\end{tabular}

The $k^{\vee}$-variables are given by

$$
\begin{aligned}
& k_{1}^{\vee}=-s_{1}(0)-s_{1}(1)+s_{2}(0)=-k_{1}+r_{2} \\
& k_{2}^{\vee}=-s_{2}(1)-s_{2}(2)+s_{1}(1)=k_{1}-k_{2}-r_{1} .
\end{aligned}
$$

Putting these into the definition of mutation weight (3.7) and summing over $k$ variables, we obtain

$$
\begin{aligned}
Z_{\boldsymbol{m}}\left(r_{1}, r_{2}\right) & =\sum_{k_{1}, k_{2} \geq 0} W\left(k_{1},-k_{1}+r_{2}\right) W\left(k_{2}, k_{1}-k_{2}-r_{1}\right) y^{\left(k_{1}, k_{2}\right)} \\
& =\sum_{k_{1}, k_{2} \geq 0} q^{\frac{1}{2}\left(k_{1}^{2}+k_{2}^{2}-k_{1} k_{2}-k_{1} r_{2}+k_{2} r_{1}\right)}\left[\begin{array}{c}
r_{2} \\
k_{1}
\end{array}\right]_{q}\left[\begin{array}{c}
k_{1}-r_{1} \\
k_{2}
\end{array}\right]_{q} y^{\left(k_{1}, k_{2}\right)} .
\end{aligned}
$$


For example,

$$
\begin{aligned}
Z_{\boldsymbol{m}}(-2,1)= & +y^{(1,0)}+\left(q^{-\frac{1}{2}}+q^{\frac{1}{2}}\right) y^{(0,1)} \\
& +\left(q^{-1}+1+q\right) y^{(1,1)}+y^{(0,2)}+\left(q^{-1}+1+q\right) y^{(1,2)}+y^{(1,3)}, \\
Z_{\boldsymbol{m}}(2,2)= & +\left(q^{-\frac{1}{2}}+q^{\frac{1}{2}}\right) y^{(1,0)}+\left(-q^{-\frac{1}{2}}-q^{\frac{1}{2}}\right) y^{(0,1)} \\
& +y^{(2,0)}+\left(-q^{-\frac{1}{2}}-q^{\frac{1}{2}}\right) y^{(1,1)}+\left(q^{-1}+1+q\right) y^{(0,2)}+\cdots .
\end{aligned}
$$

Note that the sum in $Z_{\boldsymbol{m}}(-2,1)$ is actually finite, whereas the sum in $Z_{\boldsymbol{m}}(2,2)$ is infinite.

\section{Main Results}

4.1. Statement of the main results. Our first main result relates the partition functions to the products of quantum dilogarithms

$$
\mathbb{E}(x)=\mathbb{E}(x ; q)=\prod_{n=0}^{\infty} \frac{1}{1+q^{n+\frac{1}{2}} x} .
$$

Theorem 4.1. Let $\left[x^{n}\right] F(x)$ denote the coefficient of $x^{n}$ in the series $F(x)=$ $\sum a_{n} x^{n}$. Then, for any $\beta \in \mathbb{Z}^{n}$, we have

$$
\begin{aligned}
& q^{\frac{1}{2}\langle\beta, r\rangle}\left(\left[y^{\beta}\right] Z_{\boldsymbol{m}}(r)\right) \\
& =\left[y^{\beta}\right]\left(\left(\mathbb{E}\left(y^{\alpha_{1}} ; q^{\varepsilon_{1}}\right) \cdots \mathbb{E}\left(y^{\alpha_{T}} ; q^{\varepsilon_{T}}\right)\right)^{-1}\left(\mathbb{E}\left(q^{\left\langle\alpha_{1}, r\right\rangle} y^{\alpha_{1}} ; q^{\varepsilon_{1}}\right) \cdots \mathbb{E}\left(q^{\left\langle\alpha_{T}, r\right\rangle} y^{\alpha_{T}} ; q^{\varepsilon_{T}}\right)\right)\right)
\end{aligned}
$$

where $\langle$,$\rangle is a skew symmetric bilinear form defined by (2.9).$

Thanks to Theorem 4.1 any identity between products of quantum dilogarithm series leads to that for the partition functions. In particular, applying this result to the mutation sequences defining combinatorial Donaldson-Thomas invariants, we obtain our second main result:

Theorem 4.2. If $\boldsymbol{m}$ and $\boldsymbol{m}^{\prime}$ are two mutation sequences such that there is a frozen isomorphism between $\mu_{\boldsymbol{m}}\left(Q^{\wedge}\right)$ and $\mu_{\boldsymbol{m}^{\prime}}\left(Q^{\wedge}\right)$, then we have $Z_{\boldsymbol{m}}=Z_{\boldsymbol{m}^{\prime}}$.

First we will prove Theorem 4.1 in Section 4.2. Then the proof of Theorem 4.2 will be given in Section 4.3 .

4.2. Proof of Theorem 4.1. We first rewrite the product of quantum dilogarithms in terms of $q$-binomials as follows:

Proposition 4.3. For any mutation sequence $\boldsymbol{m}=\left(m_{1}, \ldots, m_{T}\right)$ and for any $n_{1}, \ldots, n_{T} \in \mathbb{Z}$, we have

$$
\begin{aligned}
& \left(\mathbb{E}\left(y^{\alpha_{1}} ; q^{\varepsilon_{1}}\right) \cdots \mathbb{E}\left(y^{\alpha_{T}} ; q^{\varepsilon_{T}}\right)\right)^{-1}\left(\mathbb{E}\left(q^{n_{1}} y^{\alpha_{1}} ; q^{\varepsilon_{1}}\right) \cdots \mathbb{E}\left(q^{n_{T}} y^{\alpha_{T}} ; q^{\varepsilon_{T}}\right)\right) \\
& =\sum_{k \geq 0}\left(\prod_{t=1}^{T} q^{\frac{1}{2} \varepsilon_{t} k_{t}^{2}}\left[\begin{array}{c}
\varepsilon_{t}\left(n_{t}+\sum_{i=1}^{t-1}\left\langle\alpha_{i}, \alpha_{t}\right\rangle k_{i}\right) \\
k_{t}
\end{array}\right]_{q^{\varepsilon_{t}}}\right) y^{k_{T} \alpha_{T}} \cdots y^{k_{1} \alpha_{1}} .
\end{aligned}
$$


Proof. A key is to express $q$-binomial coefficients as the "ratio" of $q$-dilogarithms:

$$
\begin{aligned}
& \mathbb{E}(x ; q)^{-1} \mathbb{E}\left(q^{m} x ; q\right)= \begin{cases}\prod_{i=0}^{m-1}\left(1+q^{i+\frac{1}{2}} x\right) & (m \geq 0) \\
\prod_{i=0}^{-m-1}\left(1+q^{-i-\frac{1}{2}} x\right)^{-1} & (m<0)\end{cases} \\
& =\sum_{k \geq 0} q^{\frac{1}{2} k^{2}}\left[\begin{array}{c}
m \\
k
\end{array}\right]_{q} x^{k}
\end{aligned}
$$

by using the $q$-binomial theorem. Moreover, by substituting $q^{-1}$ for $q$ and $-m$ for $m$, we get

$$
\mathbb{E}\left(x ; q^{\varepsilon}\right)^{-1} \mathbb{E}\left(q^{m} x ; q^{\varepsilon}\right)=\sum_{k \geq 0} q^{\frac{1}{2} \varepsilon k^{2}}\left[\begin{array}{c}
\varepsilon m \\
k
\end{array}\right]_{q^{\varepsilon}} x^{k}
$$

for $\varepsilon \in\{1,-1\}$. Then the proof is by induction on the length $T$ of the mutation sequence. For $T=0$, equality is obvious because both sides of (4.2) are equal to 1 . For $T>0$, we set

$$
A=\prod_{t=1}^{T-1} q^{\frac{1}{2} \varepsilon_{t} k_{t}^{2}}\left[\begin{array}{c}
\varepsilon_{t}\left(n_{t}+\sum_{i=1}^{t-1}\left\langle\alpha_{i}, \alpha_{t}\right\rangle k_{i}\right) \\
k_{t}
\end{array}\right]_{q^{\varepsilon_{t}}} .
$$

By induction hypothesis, we have

$$
\begin{aligned}
& \left(\mathbb{E}\left(y^{\alpha_{1}} ; q^{\varepsilon_{1}}\right) \cdots \mathbb{E}\left(y^{\alpha_{T-1}} ; q^{\varepsilon_{T-1}}\right)\right)^{-1}\left(\mathbb{E}\left(q^{n_{1}} y^{\alpha_{1}} ; q^{\varepsilon_{1}}\right) \cdots \mathbb{E}\left(q^{n_{T-1}} y^{\alpha_{T-1}} ; q^{\varepsilon_{T-1}}\right)\right) \\
& =\sum_{k_{1}, \ldots k_{T-1} \geq 0} A y^{k_{T-1} \alpha_{T-1}} \cdots y^{k_{1} \alpha_{1}} .
\end{aligned}
$$

Using the commutation relation

$$
\left(y^{k_{T-1} \alpha_{T-1}} \cdots y^{k_{1} \alpha_{1}}\right) y^{\alpha_{T}}=q^{\sum_{i=1}^{T-1}\left\langle\alpha_{i}, \alpha_{T}\right\rangle k_{i}} y^{\alpha_{T}}\left(y^{k_{T-1} \alpha_{T-1}} \cdots y^{k_{1} \alpha_{1}}\right)
$$

and (4.4), we obtain

$$
\begin{aligned}
& \left(\mathbb{E}\left(y^{\alpha_{1}} ; q^{\varepsilon_{1}}\right) \cdots \mathbb{E}\left(y^{\alpha_{T}} ; q^{\varepsilon_{T}}\right)\right)^{-1}\left(\mathbb{E}\left(q^{n_{1}} y^{\alpha_{1}} ; q^{\varepsilon_{1}}\right) \cdots \mathbb{E}\left(q^{n_{T}} y^{\alpha_{T}} ; q^{\varepsilon_{T}}\right)\right) \\
& =\mathbb{E}\left(y^{\alpha_{T}} ; q^{\varepsilon_{T}}\right)^{-1}\left(\sum_{k_{1}, \ldots k_{T-1} \geq 0} A y^{k_{T-1} \alpha_{T-1}} \cdots y^{k_{1} \alpha_{1}}\right) \mathbb{E}\left(q^{n_{T}} y^{\alpha_{T}} ; q^{\varepsilon_{T}}\right) \\
& =\sum_{k_{1}, \ldots k_{T-1} \geq 0} \mathbb{E}\left(y^{\alpha_{T}} ; q^{\varepsilon_{T}}\right)^{-1} \mathbb{E}\left(q^{n_{T}+\sum_{i=1}^{T-1}\left\langle\alpha_{i}, \alpha_{T}\right\rangle k_{i}} y^{\alpha_{T}} ; q^{\varepsilon_{T}}\right) A y^{k_{T-1} \alpha_{T-1}} \cdots y^{k_{1} \alpha_{1}} \\
& \left.=\sum_{k \geq 0} q^{\frac{1}{2} \varepsilon_{T} k_{T}^{2}}\left[\begin{array}{c}
\left.\varepsilon_{T}\left(n_{T}+\sum_{i=1}^{T-1}\left\langle\alpha_{i}, \alpha_{T}\right\rangle k_{i}\right)\right]_{q_{T}^{\varepsilon_{T}}} y^{k_{T} \alpha_{T}} A y^{k_{T} \alpha_{T-1}} \cdots y^{k_{1} \alpha_{1}} \\
k_{T}
\end{array}\right]_{q^{\varepsilon_{t}}}\right) y^{k_{T} \alpha_{T} \cdots y^{k_{1} \alpha_{1}}} \\
& =\sum_{k \geq 0}\left(\prod _ { t = 1 } ^ { T } q ^ { \frac { 1 } { 2 } \varepsilon _ { t } k _ { t } ^ { 2 } } \left[\begin{array}{c}
\varepsilon_{t}\left(n_{t}+\sum_{i=1}^{t-1}\left\langle\alpha_{i}, \alpha_{t}\right\rangle k_{i}\right) \\
k_{t}
\end{array}\right.\right.
\end{aligned}
$$

This completes the proof of Proposition 4.3

We introduce the notion of a state vector considered in [10]. The state vector of $Q(t)$ is defined by

$$
\psi(t):=\sum_{i=1}^{n} s_{i}(t) c_{i}(t) \in \mathbb{Z}^{n} \quad(0 \leq t \leq T) .
$$


By the definition of $c$-vectors, we have $\psi(0)=\left(s_{1}(0), \ldots, s_{n}(0)\right)=r$.

We use the following properties shown in 10, 1 :

- For the mutation sequence (3.1), we have

$$
B(t)_{i j}=\left\langle c_{i}(t), c_{j}(t)\right\rangle, \quad(0 \leq t \leq T)
$$

or equivalently,

$$
C(t) B(0) C(t)^{\top}=B(t) . \quad(0 \leq t \leq T)
$$

- Along the mutation sequence (3.1), the state vector changes as

$$
\psi(t)=\psi(t-1)-k_{t} \alpha_{t}, \quad(t=1, \ldots, T) .
$$

- The state vectors of the initial and the final quivers are related as

$$
\psi(0)-\psi(T)=\sum_{t=1}^{T} k_{t} \alpha_{t} .
$$

- For any mutation sequence, we have

$$
-\sum_{t=1}^{T} \varepsilon_{t} k_{t} k_{t}^{\vee}+\langle\psi(0), \psi(T)\rangle=\sum_{t=1}^{T} \varepsilon_{t} k_{t}^{2}-\sum_{1 \leq i<j \leq T} k_{i} k_{j}\left\langle\alpha_{i}, \alpha_{j}\right\rangle .
$$

We also need the following:

Proposition 4.4. For any mutation sequences, we have

$$
k_{t}+k_{t}^{\vee}=\varepsilon_{t}\left(\left\langle\alpha_{t}, \psi(0)\right\rangle+\sum_{i=1}^{t-1}\left\langle\alpha_{i}, \alpha_{t}\right\rangle k_{i}\right) .
$$

Proof.

$$
\begin{aligned}
& k_{t}+k_{t}^{\vee}=\sum_{i=1}^{n} B(t-1)_{m_{t}, i} s_{i}(t-1) \\
& \text { (by (3.6)) } \\
& =\sum_{i=1}^{n}\left\langle c_{m_{t}}(t-1), c_{i}(t-1)\right\rangle s_{i}(t-1) \\
& =\left\langle c_{m_{t}}(t-1), \sum_{i=1}^{n} c_{i}(t-1) s_{i}(t-1)\right\rangle \\
& =\left\langle c_{m_{t}}(t-1), \psi(t-1)\right\rangle \\
& =\left\langle\varepsilon_{t} \alpha_{t}, \psi(t-1)\right\rangle \\
& \text { (by (3.9) ) } \\
& =\left\langle\varepsilon_{t} \alpha_{t}, \psi(0)-\sum_{i=1}^{t-1} \alpha_{i} k_{i}\right\rangle \\
& \left.=\varepsilon_{t}\left(\left\langle\alpha_{t}, \psi(0)\right\rangle+\sum_{i=1}^{t-1}\left\langle\alpha_{i}, \alpha_{t}\right\rangle k_{i}\right) . \quad \text { (by skewness of }\langle,\rangle\right)
\end{aligned}
$$

\footnotetext{
${ }^{1}$ Note that $k_{t}^{\vee}$ in this paper is $-k_{t}^{\vee}$ in [10].
} 
We are now ready to prove Theorem 4.1. The partition function associated with the mutation sequence $\boldsymbol{m}$ and initial $s$-variables $r=\left(r_{1}, \ldots, r_{n}\right)$ is given by

$$
\begin{aligned}
Z_{\boldsymbol{m}}(r) & =\sum_{k \geq 0}\left(\prod_{t=1}^{T} W^{\varepsilon_{t}}\left(k_{t}, k_{t}^{\vee}\right)\right) y^{\sum_{t=1}^{T} k_{t} \alpha_{t}} \\
& =\sum_{k \geq 0}\left(\prod_{t=1}^{T} q^{-\frac{1}{2} \varepsilon_{t} k_{t} k_{t}^{\vee}}\left[\begin{array}{c}
k_{t}+k_{t}^{\vee} \\
k_{t}
\end{array}\right]_{q^{\varepsilon_{t}}}\right) y^{\sum_{t=1}^{T} k_{t} \alpha_{t}} .
\end{aligned}
$$

On the other hand, the ratio of the quantum dilogarithms products along $\boldsymbol{m}$ is given by

$$
\begin{aligned}
& \left(\mathbb{E}\left(y^{\alpha_{1}} ; q^{\varepsilon_{1}}\right) \cdots \mathbb{E}\left(y^{\alpha_{T}} ; q^{\varepsilon_{T}}\right)\right)^{-1} \mathbb{E}\left(q^{\left\langle\alpha_{1}, r\right\rangle} y^{\alpha_{1}} ; q^{\varepsilon_{1}}\right) \cdots \mathbb{E}\left(q^{\left\langle\alpha_{T}, r\right\rangle} y^{\alpha_{T}} ; q^{\varepsilon_{T}}\right) \\
& =\sum_{k \geq 0}\left(\prod_{t=1}^{T} q^{\frac{1}{2} \varepsilon_{t} k_{t}^{2}}\left[\begin{array}{c}
\left.\varepsilon_{t}\left(\left\langle\alpha_{t}, r\right\rangle+\sum_{i=1}^{t-1}\left\langle\alpha_{i}, \alpha_{t}\right\rangle k_{i}\right)\right]_{q^{\varepsilon} t} \\
k_{t}
\end{array}\right) y^{k_{T} \alpha_{T}} \cdots y^{k_{1} \alpha_{1}}\right. \\
& =\sum_{k \geq 0}\left(\prod_{t=1}^{T} q^{\frac{1}{2} \varepsilon_{t} k_{t}^{2}}\left[\begin{array}{c}
\left.\varepsilon_{t}\left(\left\langle\alpha_{t}, \psi(0)\right\rangle+\sum_{i=1}^{t-1}\left\langle\alpha_{i}, \alpha_{t}\right\rangle k_{i}\right)\right]_{t}^{k_{T} \alpha_{T}} \cdots y^{k_{1} \alpha_{1}} \\
k_{t}
\end{array}\right]_{q^{\varepsilon_{t}}}\right) q^{-\frac{1}{2} \sum_{1 \leq i \leq j \leq T} k_{i} k_{j}\left\langle\alpha_{i}, \alpha_{j}\right\rangle} y^{\sum_{t=1}^{T} k_{t} \alpha_{t}} . \\
& =\sum_{k \geq 0}\left(\prod _ { t = 1 } ^ { T } q ^ { \frac { 1 } { 2 } \varepsilon _ { t } k _ { t } ^ { 2 } } \left[\begin{array}{c}
\varepsilon_{t}\left(\left\langle\alpha_{t}, \psi(0)\right\rangle+\sum_{i=1}^{t-1}\left\langle\alpha_{i}, \alpha_{t}\right\rangle k_{i}\right) \\
k_{t}
\end{array}\right.\right.
\end{aligned}
$$

Therefore, to prove Theorem 4.1, it suffices to show that the following equalities:

$$
\begin{gathered}
k_{t}+k_{t}^{\vee}=\varepsilon_{t}\left(\left\langle\alpha_{t}, \psi(0)\right\rangle+\sum_{i=1}^{t-1}\left\langle\alpha_{i}, \alpha_{t}\right\rangle k_{i}\right), \\
-\frac{1}{2} \sum_{t=1}^{T} \varepsilon_{t} k_{t} k_{t}^{\vee}+\frac{1}{2}\left\langle\sum_{t=1}^{T} k_{t} \alpha_{t}, r\right\rangle=\frac{1}{2} \sum_{t=1}^{T} \varepsilon_{t} k_{t}^{2}-\frac{1}{2} \sum_{1 \leq i \leq j \leq T} k_{i} k_{j}\left\langle\alpha_{i}, \alpha_{j}\right\rangle .
\end{gathered}
$$

The equality (4.12) follows immediately from Proposition 4.4. (4.13) follows from (4.10) because

$$
\left\langle\sum_{t=1}^{T} k_{t} \alpha_{t}, r\right\rangle=\langle\psi(0)-\psi(T), \psi(0)\rangle=\langle\psi(0), \psi(T)\rangle
$$

by (4.9) and the skewness of $\langle$,$\rangle . This completes the proof of Theorem 4.1$,

4.3. Proof of Theorem 4.2, We first review some known results about quantum dilogarithms. For a mutation sequence $\boldsymbol{m}=\left(m_{1}, \ldots, m_{T}\right)$ of $Q$ we consider the following ordered product of quantum dilogarithms:

$$
\mathbb{E}(Q ; \boldsymbol{m}):=\mathbb{E}\left(y^{\alpha_{1}} ; q^{\varepsilon_{1}}\right) \mathbb{E}\left(y^{\alpha_{2}} ; q^{\varepsilon_{2}}\right) \cdots \mathbb{E}\left(y^{\alpha_{T}} ; q^{\varepsilon_{T}}\right) \quad \in \widehat{\mathbb{A}}_{Q}
$$

Theorem 4.5 (Keller[12, Nagao[17]). If $\boldsymbol{m}$ and $\boldsymbol{m}^{\prime}$ are two mutation sequences such that there is a frozen isomorphism between $\mu_{\boldsymbol{m}}\left(Q^{\wedge}\right)$ and $\mu_{\boldsymbol{m}^{\prime}}\left(Q^{\wedge}\right)$, then we have $\mathbb{E}(Q ; \boldsymbol{m})=\mathbb{E}\left(Q ; \boldsymbol{m}^{\prime}\right)$.

Theorem 4.6 (Keller[12]). If $\boldsymbol{m}$ and $\boldsymbol{m}^{\prime}$ are reddening sequences on the quiver $Q$, then there is a frozen isomorphism between the final ice quivers $\mu_{m}\left(Q^{\wedge}\right) \simeq$ $\mu_{m^{\prime}}\left(Q^{\wedge}\right)$. 
Theorems 4.5 and 4.6 imply that if $Q$ admits a reddening sequence $\boldsymbol{m}$, then the power series

$$
\mathbb{E}_{Q}:=\mathbb{E}(Q ; \boldsymbol{m}) \in \widehat{\mathbb{A}}_{Q} .
$$

is independent of the choice of the reddening sequence $\boldsymbol{m}$. Thus it is intrinsically associated with the quiver $Q$. Keller 13 named this invariant as combinatorial Donaldson-Thomas (DT) invariant. Note that the statements of Theorems 4.6 is combinatorial, but the known proofs are based on categorification in terms of Ginzburg dg-algebra [6]. The well-known pentagon identity

$$
\mathbb{E}(x) \mathbb{E}(y)=\mathbb{E}(y) \mathbb{E}\left(q^{-\frac{1}{2}} x y\right) \mathbb{E}(x)
$$

for $x y=q y x$ is nothing but the combinatorial DT invariant of $A_{2}$ quiver $Q=$ $(1 \rightarrow 2)$ corresponding to the two reddening sequences $\boldsymbol{m}=(1,2)$ and $\boldsymbol{m}^{\prime}=(2,1,2)$ depicted in Figure 1 .

Thanks to the identities of quantum dilogarithms, Theorem 4.2 follows immediately from our first main result Theorem 4.1

Proof of Theorem 4.2. From Theorem 4.5] we have $\mathbb{E}(Q ; \boldsymbol{m})=\mathbb{E}\left(Q ; \boldsymbol{m}^{\prime}\right)$. This equality also holds if we replace $\left(y^{\alpha_{1}}, \ldots, y^{\alpha_{T}}\right)$ by $\left(q^{n_{1}} y^{\alpha_{1}}, \ldots, q^{n_{T}} y^{\alpha_{T}}\right)$ for $\left(n_{1}, \ldots, n_{T}\right) \in$ $\mathbb{Z}^{T}$ because they have the same commutation relations. Therefore, Theorem 4.1 implies that $Z_{m}=Z_{m^{\prime}}$.

One of the consequences is that, from a viewpoint of Theorem 4.6] if a quiver $Q$ admit a reddening sequence $\boldsymbol{m}$, our partition function $Z_{\boldsymbol{m}}$ is independent of the choice of the reddening sequence.

\section{EXAMPLES}

In this section, we show some sample computation of partition functions for some mutation sequences. We hope that these examples illustrate how our results provide a systematic way of constructing various $q$-binomial multisum identities.

Throughout this section, we simply write $W\left(k_{t}, k_{t}^{\vee}\right)$ for $W^{+}\left(k_{t}, k_{t}^{\vee}\right)$ when we consider green sequences.

Example 5.1. Consider the $A_{2}$ quiver

$$
Q=1 \longrightarrow 2
$$

and a mutation sequence $\boldsymbol{m}=(1,2)$. The partition function of $\boldsymbol{m}$ is computed in Example 3.5

$$
Z_{\boldsymbol{m}}\left(r_{1}, r_{2}\right)=\sum_{k_{1}, k_{2} \geq 0} W\left(k_{1},-k_{1}+r_{2}\right) W\left(k_{2}, k_{1}-k_{2}-r_{1}\right) y^{\left(k_{1}, k_{2}\right)} .
$$

The coefficient of $y^{\left(\beta_{1}, \beta_{2}\right)}$ in the partition function $(5.2)$ is given by

$$
\begin{aligned}
{\left[y^{\left(\beta_{1}, \beta_{2}\right)}\right] Z_{\boldsymbol{m}}\left(r_{1}, r_{2}\right) } & =W\left(\beta_{1},-\beta_{1}+r_{2}\right) W\left(\beta_{2}, \beta_{1}-\beta_{2}-r_{1}\right) \\
& =q^{\frac{1}{2}\left(\beta_{1}^{2}+\beta_{2}^{2}-\beta_{1} \beta_{2}-\beta_{1} r_{2}+\beta_{2} r_{1}\right)}\left[\begin{array}{c}
r_{2} \\
\beta_{1}
\end{array}\right]_{q}\left[\begin{array}{c}
\beta_{1}-r_{1} \\
\beta_{2}
\end{array}\right]_{q} .
\end{aligned}
$$

By Theorem 4.1 this is equal to

$$
q^{-\frac{1}{2}\left(\beta_{1} r_{2}-\beta_{2} r_{1}\right)}\left[y^{\left(\beta_{1}, \beta_{2}\right)}\right]\left(\left(\mathbb{E}\left(y_{1} ; q\right) \mathbb{E}\left(y_{2} ; q\right)\right)^{-1} \mathbb{E}\left(q^{r_{2}} y_{1} ; q\right) \mathbb{E}\left(q^{-r_{1}} y_{2} ; q\right)\right) .
$$


Example 5.2. We again consider the $A_{2}$ quiver (5.1). The mutation sequences $\boldsymbol{m}=(1,2)$ and $\boldsymbol{m}^{\prime}=(2,1,2)$ of $Q$ are maximal green sequences. In addition, the permutation of vertices $(1,2)$ is a frozen isomorphism between $\mu_{\boldsymbol{m}}\left(Q^{\vee}\right)$ and $\mu_{m^{\prime}}\left(Q^{\vee}\right)$ (see Figure 1). It follows from Theorem 4.2 that

$$
Z_{\boldsymbol{m}}\left(r_{1}, r_{2}\right)=Z_{\boldsymbol{m}^{\prime}}\left(r_{1}, r_{2}\right)
$$

for all $r_{1}, r_{2} \in \mathbb{Z}$. Let us express (5.4) in terms of mutation weight.

In the mutation sequences $\boldsymbol{m}^{\prime}$, the $s$-variables change as follows:

\begin{tabular}{c|c|c} 
& $s_{1}(t)$ & $s_{2}(t)$ \\
\hline$t=0$ & $r_{1}$ & $r_{2}$ \\
$t=1$ & $r_{1}$ & $k_{1}-r_{2}+r_{1}$ \\
$t=2$ & $k_{2}-r_{1}+s_{2}(1)$ & $k_{1}-r_{2}+r_{1}$ \\
$t=3$ & $k_{2}-r_{1}+s_{2}(1)$ & $k_{3}-s_{2}(2)+s_{1}(2)$
\end{tabular}

The $k^{\vee}$-variables are computed to be

$$
\begin{aligned}
& k_{1}^{\vee}=-s_{2}(0)-s_{2}(1)=-k_{1}-r_{1}, \\
& k_{2}^{\vee}=-s_{1}(1)-s_{1}(2)=-k_{1}-k_{2}-r_{1}+r_{2}, \\
& k_{3}^{\vee}=-s_{2}(2)-s_{2}(3)=-k_{1}-k_{2}-k_{3}+r_{2} .
\end{aligned}
$$

The partition function of $\boldsymbol{m}^{\prime}$ is given by

$$
\begin{gathered}
Z_{\boldsymbol{m}^{\prime}}\left(r_{1}, r_{2}\right)=\sum_{k_{1}, k_{2}, k_{3} \geq 0} W\left(k_{1},-k_{1}-r_{1}\right) W\left(k_{2},-k_{1}-k_{2}-r_{1}+r_{2}\right) \\
\cdot W\left(k_{3},-k_{1}-k_{2}-k_{3}+r_{2}\right) y^{\left(k_{2}+k_{3}, k_{1}+k_{2}\right)} .
\end{gathered}
$$

Comparing the coefficients of $y^{\left(\beta_{1}, \beta_{2}\right)}$ in (5.2) and (5.6) gives a five-term identity of mutation weights:

$$
\begin{aligned}
& W\left(\beta_{1},-\beta_{1}+r_{2}\right) W\left(\beta_{2}, \beta_{1}-\beta_{2}+r_{1}\right) \\
& =\sum_{\substack{k_{1}, k_{2}, k_{3} \geq 0 \\
k_{2}+k_{3}=\beta_{1} \\
k_{1}+k_{2}=\beta_{2}}} W\left(k_{1},-k_{1}-r_{1}\right) W\left(k_{2},-\beta_{2}-r_{1}+r_{2}\right) W\left(k_{3},-\beta_{2}-k_{3}+r_{2}\right) .
\end{aligned}
$$

Multiplying both sides by $q^{-\frac{1}{2}\left(\beta_{1}^{2}+\beta_{2}^{2}-\beta_{1} \beta_{2}-\beta_{1} r_{2}+\beta_{2} r_{1}\right)}$, we get a five-term $q$-binomial identity:

$$
\left[\begin{array}{c}
r_{2} \\
\beta_{1}
\end{array}\right]_{q}\left[\begin{array}{c}
\beta_{1}-r_{1} \\
\beta_{2}
\end{array}\right]_{q}=\sum_{\substack{k_{1}, k_{2}, k_{3} \geq 0 \\
k_{2}+k_{3}=\beta_{1} \\
k_{1}+k_{2}=\beta_{2}}} q^{k_{1} k_{3}}\left[\begin{array}{c}
-r_{1} \\
k_{1}
\end{array}\right]_{q}\left[\begin{array}{c}
-r_{1}+r_{2}-k_{1} \\
k_{2}
\end{array}\right]_{q}\left[\begin{array}{c}
-\beta_{2}+r_{2} \\
k_{3}
\end{array}\right]_{q}
$$

Remark 5.3. This identity is equivalent to the Stanley's identity (see [7])

$$
\left[\begin{array}{c}
c+a \\
a
\end{array}\right]_{q}\left[\begin{array}{c}
d+b \\
b
\end{array}\right]_{q}=\sum_{k=0}^{\min (a, b)} q^{(a-k)(b-k)}\left[\begin{array}{c}
c+d+k \\
k
\end{array}\right]_{q}\left[\begin{array}{c}
c+a-b \\
a-k
\end{array}\right]_{q}\left[\begin{array}{c}
d+b-a \\
b-k
\end{array}\right]_{q} .
$$

Example 5.4. Consider an alternating quiver of type $A_{3}$

$$
Q=1 \longrightarrow 2 \longleftarrow 3
$$




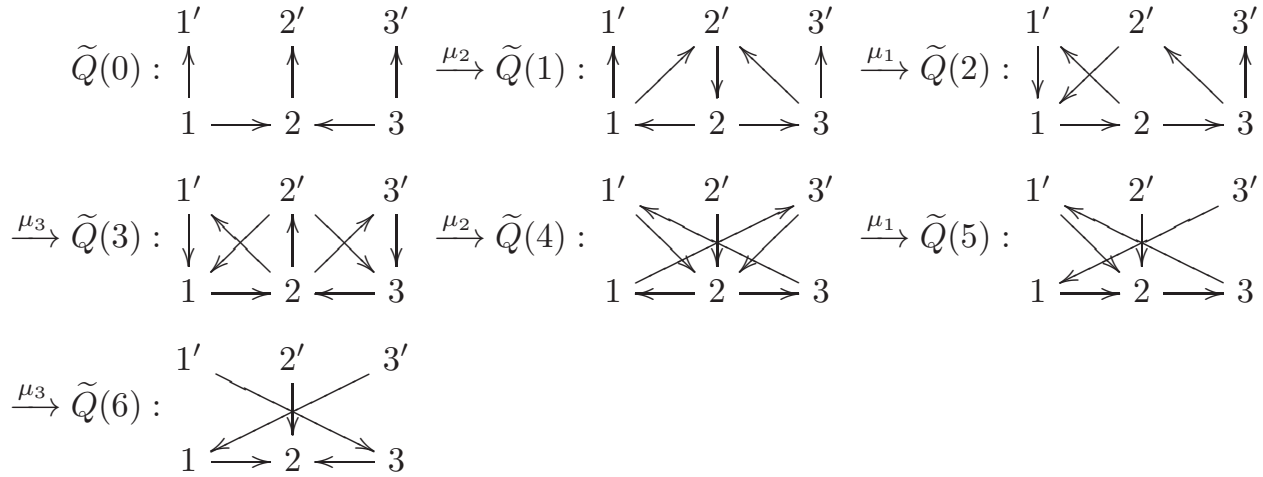

Figure 2. A mutation sequence $\boldsymbol{m}^{\prime}=(2,1,3,2,1,3)$ for an $A_{3}$ quiver.

The mutation sequences $\boldsymbol{m}=(1,3,2)$ and $\boldsymbol{m}^{\prime}=(2,1,3,2,1,3)$ of $Q$ are maximal green sequences. In addition, the permutation of vertices $(1,3)$ is a frozen isomorphism between $\mu_{\boldsymbol{m}}\left(Q^{\vee}\right)$ and $\mu_{\boldsymbol{m}^{\prime}}\left(Q^{\vee}\right)$ (see Figure 5.4). It follows from Theorem 4.2 that

$$
Z_{\boldsymbol{m}}\left(r_{1}, r_{2}, r_{3}\right)=Z_{\boldsymbol{m}^{\prime}}\left(r_{1}, r_{2}, r_{3}\right)
$$

for all $r_{1}, r_{2}, r_{3} \in \mathbb{Z}$. Let us write (5.11) in terms of mutation weights.

In the mutation sequences $\boldsymbol{m}$, the $s$-variables change as follows:

\begin{tabular}{c|c|c|c} 
& $s_{1}(t)$ & $s_{2}(t)$ & $s_{3}(t)$ \\
\hline$t=0$ & $r_{1}$ & $r_{2}$ & $r_{3}$ \\
$t=1$ & $k_{1}-r_{1}$ & $r_{2}$ & $r_{3}$ \\
$t=2$ & $k_{1}-r_{1}$ & $r_{2}$ & $k_{2}-r_{3}$ \\
$t=3$ & $k_{1}-r_{1}$ & $k_{3}-r_{2}$ & $k_{2}-r_{3}$
\end{tabular}

The $k^{\vee}$-variables are computed to be

$$
\begin{aligned}
& k_{1}^{\vee}=-s_{1}(0)-s_{1}(1)+s_{2}(0)=-k_{1}+r_{2} \\
& k_{2}^{\vee}=-s_{3}(1)-s_{3}(2)+s_{2}(1)=-k_{2}+r_{2} \\
& k_{3}^{\vee}=-s_{2}(2)-s_{2}(3)+s_{1}(2)+s_{3}(2)=k_{1}+k_{2}-k_{3}-r_{1}-r_{3} .
\end{aligned}
$$

The partition function of $\boldsymbol{m}$ is given by

$$
\begin{aligned}
& Z_{\boldsymbol{m}}\left(r_{1}, r_{2}, r_{3}\right)=\sum_{k_{1}, k_{2}, k_{3} \geq 0} W\left(k_{1},-k_{1}+r_{2}\right) \\
& \cdot W\left(k_{2},-k_{2}+r_{2}\right) W\left(k_{3},-k_{3}+k_{1}+k_{2}-r_{1}-r_{3}\right) y^{\left(k_{1}, k_{3}, k_{2}\right)} .
\end{aligned}
$$


On the other hand, in the mutation sequences $\boldsymbol{m}^{\prime}$, the $s$-variables change as follows:

\begin{tabular}{c|c|c|c} 
& $s_{1}(t)$ & $s_{2}(t)$ & $s_{3}(t)$ \\
\hline$t=0$ & $r_{1}$ & $r_{2}$ & $r_{3}$ \\
$t=1$ & $r_{1}$ & $k_{1}-r_{2}+r_{1}+r_{3}$ & $r_{3}$ \\
$t=2$ & $k_{2}-r_{1}+s_{2}(1)$ & $k_{1}-r_{2}+r_{1}+r_{3}$ & $r_{3}$ \\
$t=3$ & $k_{2}-r_{1}+s_{2}(1)$ & $k_{1}-r_{2}+r_{1}+r_{3}$ & $k_{3}-r_{3}+s_{2}(2)$ \\
$t=4$ & $k_{2}-r_{1}+s_{2}(1)$ & $k_{4}-s_{2}(3)+s_{1}(3)+s_{3}(3)$ & $k_{3}-r_{3}+s_{2}(2)$ \\
$t=5$ & $k_{5}-s_{1}(4)+s_{2}(4)$ & $k_{4}-s_{2}(3)+s_{1}(3)+s_{3}(3)$ & $k_{3}-r_{3}+s_{2}(2)$ \\
$t=6$ & $k_{5}-s_{1}(4)+s_{2}(4)$ & $k_{4}-s_{2}(3)+s_{1}(3)+s_{3}(3)$ & $k_{6}-s_{3}+s_{2}(5)$
\end{tabular}

The $k^{\vee}$-variables are computed to be

$$
\begin{aligned}
& k_{1}^{\vee}=-s_{2}(0)-s_{2}(1)=-k_{1}-r_{1}-r_{3}, \\
& k_{2}^{\vee}=-s_{1}(1)-s_{1}(2)=-k_{1}-k_{2}-r_{1}+r_{2}-r_{3}, \\
& k_{3}^{\vee}=-s_{3}(2)-s_{3}(3)=-k_{1}-k_{3}-r_{1}+r_{2}-r_{3}, \\
& k_{4}^{\vee}=-s_{2}(3)-s_{2}(4)=-2 k_{1}-k_{2}-k_{3}-k_{4}-r_{1}+2 r_{2}-r_{3}, \\
& k_{5}^{\vee}=-s_{1}(4)-s_{1}(5)=-k_{1}-k_{2}-k_{3}-k_{4}-k_{5}+r_{2}, \\
& k_{6}^{\vee}=-s_{3}(5)-s_{3}(6)=-k_{1}-k_{2}-k_{3}-k_{4}-k_{6}+r_{2} .
\end{aligned}
$$

The partition function of $\boldsymbol{m}^{\prime}$ is given by

$$
\begin{aligned}
& Z_{\boldsymbol{m}^{\prime}}\left(r_{1}, r_{2}, r_{3}\right)=\sum_{k_{1}, \ldots, k_{6} \geq 0} W\left(k_{1},-k_{1}-r_{1}-r_{3}\right) W\left(k_{2},-k_{1}-k_{2}-r_{1}+r_{2}-r_{3}\right) \\
& \cdot W\left(k_{3},-k_{1}-k_{3}-r_{1}+r_{2}-r_{3}\right) W\left(k_{4},-2 k_{1}-k_{2}-k_{3}-k_{4}-r_{1}+2 r_{2}-r_{3}\right) \\
& \cdot W\left(k_{5},-k_{1}-k_{2}-k_{3}-k_{4}-k_{5}+r_{2}\right) W\left(k_{6},-k_{1}-k_{2}-k_{3}-k_{4}-k_{6}+r_{2}\right) \\
& \cdot y^{\left(k_{2}+k_{4}+k_{6}, k_{1}+k_{2}+k_{3}+k_{4}, k_{3}+k_{4}+k_{5}\right)} .
\end{aligned}
$$

Comparing the coefficients of $y^{\left(\beta_{1}, \beta_{2}, \beta_{3}\right)}$ in $Z_{\boldsymbol{m}}$ and $Z_{\boldsymbol{m}^{\prime}}$ gives a nine-term identity of mutation weights:

$$
\begin{aligned}
& W\left(\beta_{1},-\beta_{1}+r_{2}\right) W\left(\beta_{3},-\beta_{3}+r_{2}\right) W\left(\beta_{2}, \beta_{1}-\beta_{2}+\beta_{3}-r_{1}-r_{3}\right) \\
& =\sum_{k_{1}, \ldots, k_{6} \geq 0,(*)} W\left(k_{1},-k_{1}-r_{1}-r_{3}\right) W\left(k_{2},-k_{1}-k_{2}-r_{1}+r_{2}-r_{3}\right) \\
& \quad \cdot W\left(k_{3},-k_{1}-k_{3}-r_{1}+r_{2}-r_{3}\right) W\left(k_{4},-k_{1}-\beta_{2}-r_{1}+2 r_{2}-r_{3}\right) \\
& \quad \cdot W\left(k_{5},-k_{5}-\beta_{2}+r_{2}\right) W\left(k_{6},-k_{6}-\beta_{2}+r_{2}\right),
\end{aligned}
$$

where $(*)$ is the following conditions:

$$
(*) \Leftrightarrow\left\{\begin{array}{l}
k_{2}+k_{4}+k_{6}=\beta_{1} \\
k_{1}+k_{2}+k_{3}+k_{4}=\beta_{2} \\
k_{3}+k_{4}+k_{5}=\beta_{3}
\end{array} .\right.
$$


This gives the following identity of $q$-binomial coefficients:

$$
\begin{aligned}
{\left[\begin{array}{l}
r_{2} \\
\beta_{1}
\end{array}\right]_{q}\left[\begin{array}{c}
r_{2} \\
\beta_{3}
\end{array}\right]_{q}\left[\begin{array}{c}
\beta_{1}+\beta_{3}-r_{1}-r_{3} \\
\beta_{2}
\end{array}\right]_{q} } \\
=\sum_{k_{1}, \ldots, k_{6} \geq 0,(*)} q^{k_{1} k_{4}+k_{1} k_{5}+k_{1} k_{6}+k_{2} k_{5}+k_{3} k_{6}}\left[\begin{array}{c}
-r_{1}-r_{3} \\
k_{1}
\end{array}\right]_{q} \\
\cdot\left[\begin{array}{c}
-k_{1}-r_{1}+r_{2}-r_{3} \\
k_{2}
\end{array}\right]_{q}\left[\begin{array}{c}
-k_{1}-r_{1}+r_{2}-r_{3} \\
k_{3}
\end{array}\right]_{q} \\
\cdot\left[\begin{array}{c}
-k_{1}+k_{4}-\beta_{2}-r_{1}+2 r_{2}-r_{3} \\
k_{4}
\end{array}\right]_{q}\left[\begin{array}{c}
-\beta_{2}+r_{2} \\
k_{5}
\end{array}\right]_{q}\left[\begin{array}{c}
-\beta_{2}+r_{2} \\
k_{6}
\end{array}\right]_{q}
\end{aligned}
$$

For example, if we set $\left(\beta_{1}, \beta_{2}, \beta_{3}\right)=(1,1,2)$ and $\left(r_{1}, r_{2}, r_{3}\right)=(0,6,-2)$, (5.18) gives

$$
\begin{aligned}
{\left[\begin{array}{l}
6 \\
1
\end{array}\right]_{q}\left[\begin{array}{l}
6 \\
2
\end{array}\right]_{q}\left[\begin{array}{l}
5 \\
1
\end{array}\right]_{q}=} & {\left[\begin{array}{l}
2 \\
0
\end{array}\right]_{q}\left[\begin{array}{l}
8 \\
0
\end{array}\right]_{q}\left[\begin{array}{l}
8 \\
0
\end{array}\right]_{q}\left[\begin{array}{c}
14 \\
1
\end{array}\right]_{q}\left[\begin{array}{l}
5 \\
1
\end{array}\right]_{q}\left[\begin{array}{l}
5 \\
0
\end{array}\right]_{q}+q\left[\begin{array}{l}
2 \\
0
\end{array}\right]_{q}\left[\begin{array}{l}
8 \\
0
\end{array}\right]_{q}\left[\begin{array}{l}
8 \\
1
\end{array}\right]_{q}\left[\begin{array}{c}
13 \\
0
\end{array}\right]_{q}\left[\begin{array}{c}
5 \\
1
\end{array}\right]_{q}\left[\begin{array}{l}
5 \\
1
\end{array}\right]_{q} } \\
& +q^{2}\left[\begin{array}{l}
2 \\
0
\end{array}\right]_{q}\left[\begin{array}{l}
8 \\
1
\end{array}\right]_{q}\left[\begin{array}{l}
8 \\
0
\end{array}\right]_{q}\left[\begin{array}{c}
13 \\
0
\end{array}\right]_{q}\left[\begin{array}{l}
5 \\
2
\end{array}\right]_{q}\left[\begin{array}{l}
5 \\
0
\end{array}\right]_{q}+q^{3}\left[\begin{array}{l}
2 \\
1
\end{array}\right]_{q}\left[\begin{array}{l}
7 \\
0
\end{array}\right]_{q}\left[\begin{array}{c}
7 \\
0
\end{array}\right]_{q}\left[\begin{array}{c}
12 \\
0
\end{array}\right]_{q}\left[\begin{array}{l}
5 \\
2
\end{array}\right]_{q}\left[\begin{array}{l}
5 \\
1
\end{array}\right]_{q} .
\end{aligned}
$$

In fact, we can check both sides are equal to

$$
\begin{gathered}
1+3 q+7 q^{2}+13 q^{3}+22 q^{4}+32 q^{5}+42 q^{6}+50 q^{7}+55 q^{8}+55 q^{9} \\
+50 q^{10}+42 q^{11}+32 q^{12}+22 q^{13}+13 q^{14}+7 q^{15}+3 q^{16}+q^{17} .
\end{gathered}
$$

Example 5.5. We give a example which has negative sign mutations. Let $Q$ be a quiver given by

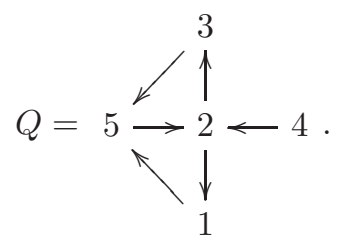

We can check directly that both of two mutation sequences

$$
\begin{aligned}
\boldsymbol{m} & =(1,3,4,2,1,3,5,2), \\
\boldsymbol{m}^{\prime} & =(2,1,3,5,2,1,3,4,2,1,3,5)
\end{aligned}
$$

are reddening sequences. The signs $\varepsilon=\left(\varepsilon_{1}^{\vee}, \ldots, \varepsilon_{8}^{\vee}\right)$ of $\boldsymbol{m}$ and $\varepsilon^{\prime}=\left(\varepsilon_{1}^{\prime \vee}, \ldots, \varepsilon_{12}^{\prime \vee}\right)$ of $\boldsymbol{m}^{\prime}$ are

$$
\begin{aligned}
\varepsilon & =(+,+,+,+,+,+,+,+), \\
\varepsilon^{\prime} & =(+,+,+,+,+,-,-,+,+,+,+,+) .
\end{aligned}
$$

The mutation sequence $\boldsymbol{m}^{\prime}$ is a example of a reddening sequence that is not a maximal green sequence.

Furthermore,

$$
\left(\begin{array}{ccccc}
1 & 2 & 3 & 4 & 5 \\
3 & 2 & 1 & 5 & 4
\end{array}\right) \in S_{5}
$$


gives a frozen isomorphism between $\mu_{m}\left(Q^{\wedge}\right)$ and $\mu_{m^{\prime}}\left(Q^{\wedge}\right)$. These mutation sequences appear in periodicity of a level 2 restricted $T$ and $Y$-system of $B_{2}[8]$. We get

$$
Z_{\boldsymbol{m}}\left(r_{1}, \ldots, r_{5}\right)=Z_{\boldsymbol{m}^{\prime}}\left(r_{1}, \ldots, r_{5}\right)
$$

by Theorem 4.2. Comparing the coefficients of $y^{\left(\beta_{1}, \ldots, \beta_{5}\right)}$ of both sides of (5.19) gives a twenty terms identity of the mutation weights

$$
\sum_{k \geq 0,(*)} W^{\varepsilon_{1}}\left(k_{1}, k_{1}^{\vee}\right) \cdots W^{\varepsilon_{8}}\left(k_{8}, k_{8}^{\vee}\right)=\sum_{k^{\prime} \geq 0,(*)^{\prime}} W^{\varepsilon_{1}^{\prime}}\left(k_{1}^{\prime}, k_{1}^{\prime \vee}\right) \cdots W^{\varepsilon_{12}^{\prime}}\left(k_{12}^{\prime}, k_{12}^{\prime \vee}\right)
$$

where the $k^{\vee}$-variables $k_{1}^{\vee}, \ldots, k_{8}^{\vee}$ and $k_{1}^{\prime \vee}, \ldots, k_{12}^{\prime \vee}$ are computed to be

$$
\begin{aligned}
& k_{1}^{\vee}=-k_{1}-r_{2}+r_{5}, \\
& k_{2}^{\vee}=-k_{2}-r_{2}+r_{5}, \\
& k_{3}^{\vee}=-k_{3}+r_{2}, \\
& k_{4}^{\vee}=-k_{1}-k_{2}+k_{3}-k_{4}+r_{1}-2 r_{2}+r_{3}-r_{4}+r_{5}, \\
& k_{5}^{\vee}=-k_{1}-k_{2}+k_{3}-k_{4}-k_{5}+r_{1}-r_{2}+r_{3}-r_{4}, \\
& k_{6}^{\vee}=-k_{1}-k_{2}+k_{3}-k_{4}-k_{6}+r_{1}-r_{2}+r_{3}-r_{4}, \\
& k_{7}^{\vee}=k_{1}+k_{2}+k_{4}-k_{7}-r_{1}+r_{2}-r_{3}, \\
& k_{8}^{\vee}=-k_{1}-k_{2}+k_{3}-2 k_{4}-k_{5}-k_{6}+k_{7}-k_{8}+r_{1}+r_{3}-r_{4}-r_{5},
\end{aligned}
$$

and

$$
\begin{aligned}
& k_{1}^{\prime \vee}=-k_{1}^{\prime}+r_{1}+r_{3}-r_{4}-r_{5}, \\
& k_{2}^{\prime \vee}=k_{1}^{\prime}-k_{2}^{\prime}-r_{2}+r_{5}, \\
& k_{3}^{\prime \vee}=k_{1}^{\prime}-k_{3}^{\prime}-r_{2}+r_{5}, \\
& k_{4}^{\prime \vee}=-k_{1}^{\prime}-k_{4}^{\prime}+r_{2}-r_{4}-r_{5}, \\
& k_{5}^{\prime \vee}=-k_{1}^{\prime}+k_{2}^{\prime}+k_{3}^{\prime}-k_{4}^{\prime}-k_{5}^{\prime}-r_{1}+r_{2}-r_{3}, \\
& k_{6}^{\prime \vee}=-k_{1}^{\prime}+k_{5}^{\prime}-k_{6}^{\prime}+r_{2}-r_{5}, \\
& k_{7}^{\prime \vee}=-k_{1}^{\prime}+k_{5}^{\prime}-k_{7}^{\prime}+r_{2}-r_{5}, \\
& k_{8}^{\prime \vee}=-k_{4}^{\prime}-k_{5}^{\prime}-k_{8}^{\prime}-r_{4}+r_{5}, \\
& k_{9}^{\prime \vee}=-k_{1}^{\prime}-k_{2}^{\prime}-k_{3}^{\prime}+k_{5}^{\prime}-k_{6}^{\prime}-k_{7}^{\prime}-k_{8}^{\prime}-k_{9}^{\prime}+r_{1}+r_{2}+r_{3}-r_{4}-r_{5}, \\
& k_{10}^{\prime \vee}=k_{1}^{\prime}-k_{5}^{\prime}+k_{9}^{\prime}-k_{10}^{\prime}-r_{2}+r_{5}, \\
& k_{11}^{\prime \vee}=k_{1}^{\prime}-k_{5}^{\prime}+k_{9}^{\prime}-k_{11}^{\prime}-r_{2}+r_{5}, \\
& k_{12}^{\prime \vee}=-k_{1}^{\prime}-k_{4}^{\prime}-k_{8}^{\prime}-k_{9}^{\prime}-k_{12}^{\prime}+r_{2} .
\end{aligned}
$$


In addition, $(*)$ and $(*)^{\prime}$ are the following conditions:

$$
\begin{gathered}
(*) \Leftrightarrow\left\{\begin{array}{l}
k_{1}+k_{4}+k_{6}=\beta_{1} \\
k_{4}+k_{5}+k_{6}+k_{8}=\beta_{2} \\
k_{2}+k_{4}+k_{5}=\beta_{3} \\
k_{3}=\beta_{4} \\
k_{7}=\beta_{5}
\end{array}\right. \\
(*)^{\prime} \Leftrightarrow\left\{\begin{array}{l}
k_{2}^{\prime}+k_{6}^{\prime}+k_{8}^{\prime}+k_{10}^{\prime}=\beta_{1} \\
k_{1}^{\prime}+k_{4}^{\prime}+k_{8}^{\prime}+k_{9}^{\prime}=\beta_{2} \\
k_{3}^{\prime}+k_{7}^{\prime}+k_{8}^{\prime}+k_{11}^{\prime}=\beta_{3} \\
k_{8}^{\prime}+k_{9}^{\prime}+k_{12}^{\prime}=\beta_{4} \\
k_{4}^{\prime}+k_{5}^{\prime}+k_{8}^{\prime}=\beta_{5}
\end{array}\right.
\end{gathered}
$$

\section{REFERENCES}

[1] Thomas Brüstle, Grégoire Dupont, and Matthieu Pérotin. On maximal green sequences. International Mathematics Research Notices, 2014(16):4547-4586, 2014.

[2] Harm Derksen, Jerzy Weyman, and Andrei Zelevinsky. Quivers with potentials and their representations II: Applications to cluster algebras. Journal of the American Mathematical Society, 23(3):749-790, 2010.

[3] Sergey Fomin and Andrei Zelevinsky. Cluster algebras I: foundations. Journal of the American Mathematical Society, 15(2):497-529, 2002.

[4] Sergey Fomin and Andrei Zelevinsky. $Y$-systems and generalized associahedra. Ann. of Math. (2) 158 (2003), no. 3, 977-1018.

[5] Sergey Fomin and Andrei Zelevinsky. Cluster algebras IV: coefficients. Compositio Mathematica, 143(01):112-164, 2007.

[6] Victor Ginzburg. Calabi-Yau algebras. arXiv preprint math/0612139, 2006.

[7] Henry W. Gould. A new symmetrical combinatorial ldentity. Journal of Combinatorial Theory, Series A, 13(2):278-286, 1972.

[8] Rei Inoue, Osamu Iyama, Bernhard Keller, Atsuo Kuniba, and Tomoki Nakanishi. Periodicities of $T$-systems and $Y$-systems, dilogarithm identities, and cluster algebras I: Type $B_{r}$. Publications of the Research Institute for Mathematical Sciences, 49(1):1-42, 2013.

[9] Akishi Kato and Yuji Terashima. Quiver Mutation Loops and Partition $q$-Series. Communications in Mathematical Physics, 336(2):811-830, 2015.

[10] Akishi Kato and Yuji Terashima. Quantum dilogarithms and partition $q$-series. Communications in Mathematical Physics, 338(1):457-481, 2015.

[11] Bernhard Keller. On cluster theory and quantum dilogarithm identities. In Representations of Algebras and Related Topics, Editors A. Skowronski and K. Yamagata, EMS Series of Congress Reports, European Mathematical Society, pages 85-11, 2011.

[12] Bernhard Keller. Cluster algebras and derived categories. In Derived categories in algebraic geometry, EMS Ser. Congr. Rep., pages 123-183. Eur. Math. Soc., Zürich, 2012.

[13] Bernhard Keller. Quiver mutation and combinatorial DT-invariants. FPSAC '13 The 25th International Conference on Formal Power Series and Algebraic Combinatorics. Paris, France, June 24-28, 2013, 2013.

[14] Bernhard Keller. The periodicity conjecture for pairs of Dynkin diagrams. Ann. of Math. (2) 177 (2013), no. 1, 111-170.

[15] Maxim Kontsevich and Yan Soibelman. Stability structures, motivic Donaldson-Thomas invariants and cluster transformations. arXiv preprint arXiv:0811.2435

[16] Maxim Kontsevich and Yan Soibelman. Cohomological Hall algebra, exponential Hodge structures and motivic Donaldson-Thomas invariants. Commun. Number Theory Phys. 5 (2011), no. 2, 231-352.

[17] Kentaro Nagao. Quantum dilogarithm identities. In Infinite analysis 2010-Developments in quantum integrable systems, RIMS Kôkyûroku Bessatsu, B28, pages 165-170. Res. Inst. Math. Sci. (RIMS), Kyoto, 2011. 
[18] Kentaro Nagao. Donaldson-Thomas theory and cluster algebras. Duke Mathematical Journal, 162(7):1313-1367, 2013.

[19] Tomoki Nakanishi. Dilogarithm identities for conformal field theories and cluster algebras: simply laced case. Nagoya Math. J. 202 (2011), 23-43.

[20] Tomoki Nakanishi. Periodicities in cluster algebras and dilogarithm identities. Representations of algebras and related topics, 407-443, EMS Ser. Congr. Rep., Eur. Math. Soc., Zürich, 2011.

[21] Pierre-Guy Plamondon. Cluster characters for cluster categories with infinite-dimensional morphism spaces. Advances in Mathematics, 227(1):1-39, 2011.

[22] Markus Reineke. Poisson automorphisms and quiver moduli. Journal of the Institute of Mathematics of Jussieu, 9(03):653-667, 2010.

[23] Markus Reineke. Cohomology of quiver moduli, functional equations, and integrality of Donaldson-Thomas type invariants. Compositio Mathematica, 147(03):943-964, 2011.

Graduate School of Mathematical Sciences, The University of Tokyo, 3-8-1 Komaba, Meguro-Ku, TOKYO 153-8914, JaPAN.

E-mail address: akishi@ms.u-tokyo.ac.jp

Department of Mathematical and Computing Science, Tokyo Institute of TechnolOgy, 2-12-1 OOKayama, Meguro-Ku, TOKyo 152-8550, Japan.

E-mail address: mizuno.y.aj@m.titech.ac.jp

Department of Mathematical and Computing Science, Tokyo Institute of Technology, 2-12-1 Ookayama, Meguro-Ku, TOKyo 152-8550, Japan.

E-mail address: tera@is.titech.ac.jp 